\title{
VECTOR CORRELATIONS IN MOLECULAR PHOTOFRAGMENTATIONS
}

\author{
F. J. COMES \\ Institut für Physikalische und Theoretische Chemie, \\ Universität Frankfurt am Main, Germany
}

Photofragmentation spectroscopy-the study of "half collisions" with polarized light of subdoppler line width-opens a window to look into the structure of molecules. The energy partitioning among the particular degrees of freedom of the products of the fragmentation reaction is described by the scalar properties, the direction and magnitude of a particular type of motion is described by the vector properties. The measurement of the scalar and vector properties allows a pictorial view of the intermediate state. The forces which make the fragments fly apart or rotate and vibrate can be "seen" from the line shapes. Information on the unstable intermediate state is gained from the stable fragments long after the dissociation of the parent molecule. In particular, information on the "lifetime" of the intermediate on a femtosecond time scale can be obtained.

A number of molecules, mainly three and four atomic, have been studied by this technique. Hydrogen peroxide has shown up as a textbook example. A complete analysis was possible including not only correlation of different types of fragment motion but also a correlation of the two coincident particles formed from the same parent molecule. The experimental results are in full agreement with recent calculations of the dynamics of the fragmentation on newly obtained potential energy surfaces. Hydrogen peroxide shows a strong dependence of its potential energy on the dihedral angle in the two electronic states amenable to laser excitation. This experiment further demonstrates that an analysis is also possible if two states are excited simultaneously.

Another good example is the fragmentation of hydrazoic acid for which also coincident pair correlation has been treated. Here again the results agree excellently with a qualitative picture which can be drawn from recently calculated ab initio potential energy surfaces. The $\mathrm{HN}_{3}$ example is much more complicated than the former one due to its higher structured upper potential energy surface. Strong rotational excitation is observed in the $\mathrm{N}_{2}$ fragment leaving the $\mathrm{NH}$ fragment rotationally cold.

The treatment of vector correlations in molecular photofragmentation is a powerful tool for the study of the dynamics of molecular dissociation reactions.

KEY WORDS: Photofragmentations, correlations.

\section{INTRODUCTION}

Atoms and molecules interact with each other which leads to different phenomena in molecular physics as for instance condensation or chemical transformation. The forces between the particles not only determine the strength of the interaction but they also are responsible for the structure which results from this interaction. Molecules can be weakly bound or strongly bound, polyatomic ones can be planar or non planar.

Structural information is already obtained from bulk properties. Solid material 
can be crystalline or amorphous, liquids can mix with each other in any composition or do not, gases behave perfect or can be liquefied at low temperatures. We already can deduce from its molecular heat that water molecules are nonlinear. (As a result of the equipartition of energy among the accessible molecular degrees of freedom we conclude that, besides the three translational degrees of freedom, there exist again three further degrees of freedom which belong to rotation showing unambiguously $\mathrm{H}_{2} \mathrm{O}$ to be a non linear molecule. This same argument proves $\mathrm{CO}_{2}$ to be linear.) The formation of associates or clusters in liquids is already reflected from the positive or negative heat of mixing.

The interaction between atoms and molecules forming larger molecular structures is reflected by the potential energy surface. The depth of the potential well is a measure of the binding energy, its location a measure of the equilibrium interparticle distance. Vibrations about this equilibrium position reflect the shape of the potential well. The study of vibrational spectroscopy is therefore a source of information on the ground state potential. Electronic transitions which connect the ground state with the respective excited state allow to study the "upper" potential energy surface.

A chemical reaction is the result of a transition from an initial state of the reacting system to the final product state. In a certain approximation this transition is represented by an intermediate state of characterististic lifetime. The study of the dynamics of short lived intermediates is therefore thought to be a key experiment to understand chemical reactions. Photofragmentation is a way to study short lived intermediate states. This has led in the past to characterize photodissociation as the study of "half collisions."

The photo energy, $h v$, absorbed in a photodissociation process (1)

$$
\mathrm{ABCD}+h v \longrightarrow \mathrm{AB}+\mathrm{CD}
$$

leads to an excess energy, $E_{\text {exc }}$,

$$
E_{\mathrm{exc}}=E_{h v}+E_{\text {int }}(\mathrm{ABCD})-E_{\text {Diss }}
$$

which is partitioned among the different degrees of freedom of the dissociation products

$$
E_{\text {exc }}=E_{\text {int }}(\mathrm{AB})+E_{\text {int }}(\mathrm{CD})+E_{\text {kin }}(\mathrm{AB})+E_{\text {kin }}(\mathrm{CD})
$$

The determination of the fragment energies - the scalar properties-gives already a detailed information on the fragmentation dynamics, pointing to a more direct type of reaction if the energy distribution among the products is very specific. As the kinetic energy is coupled to the velocity of the fragments or the rotational energy to their angular momenta, vector properties are another objective of study. If a fragmentation is very specific, then the fragment velocity vector or its angular momentum vector may show a distinct distribution in the molecular frame. The study of vector properties is therefore a means to look deeper into the structure of the intermediate state and to describe the forces which make the fragments fly in a specific direction or rotate about a specific axis.

It is obvious that the fragments have to be detected and to be spectroscopically characterized before they undergo collisions. This can be achieved by the use of the 
pump and probe technique with nanosecond time resolution at pressures in the mtorr range. As long as there exists no spatial anisotropy the fragment kinetic energy can be directly extracted from the Doppler width of a suitable fragment absorption line.

If the molecules are aligned in space, then the directional character of the velocity is an important source of information: the fragments will always move in a plane which contains the bond. Triatomic separating, e.g. into a diatomic and an atom, will always leave the fragment velocity vectors in the molecular plane with the angular momentum vector of the rotating diatomics vertical to it. $\vec{v}$ and $\vec{J}$ of the diatomic product are correlated vertically to each other, $\vec{v} \perp \vec{J}$. A four atomic molecule, separating e.g. into two diatomics, can give rise to a different situation. A torsional motion between the fragments will result in a parallel correlation, $\vec{v} \| \vec{J}$.

If in the reverse case a fragmentation results in a parallel correlation, $\vec{v} \| \overrightarrow{\mathbf{J}}$, then we may draw the conclusion that a torsional motion is involved in the dissociation process. In this case we make use of the possibility to align molecules in space by the excitation process which leads to dissociation. This can easily be achieved by the use of linearly polarized light. $\vec{E}$, the electric field vector of the electromagnetic wave, is a characterisitc quantity in the laboratory frame. Molecules which absorb this radiation by electric dipole transition are characterized by their transition dipole moment vector $\vec{\mu}$ which is a characteristic quantity in the molecular frame because this vector is coupled to the geometry of the molecule. The absorption of light couples the two coordinate systems with each other through the absorption cross section

$$
\sigma_{\mathrm{abs}} \sim(\vec{E} \cdot \vec{\mu})^{2}
$$

A maximum cross section exists for $\vec{E} \| \vec{\mu}$. For $\vec{E} \perp \vec{\mu}$ the absorption cross section vanishes. As in a bulk experiment the molecules are isotropically distributed in space, the absorption of light will form a subset of excited molecules being aligned in space.

The correlation of the transition dipole moment $\vec{\mu}$ with the fragment velocity vector $\vec{v},\langle\vec{\mu} \vec{v}\rangle$, or with the fragment angular momentum vector $\overrightarrow{\mathbf{J}},\langle\vec{\mu} \overrightarrow{\mathbf{J}}\rangle$, is reflected by the fragment absorption lines. Detecting the fragment in a direction vertical to the velocity direction results in a vanishing Doppler shift, whereas its detection in the direction of $\vec{v}$ will show a maximum Doppler shift. This information mation from the $\langle\vec{\mu} \vec{v}\rangle$ correlation is illustrated for a molecule of type $\mathrm{X}_{2} \mathrm{Y}_{2}$ in Figure 1a together with the different line shapes and the intensity relation. (For sake of simplicity the following assumptions are made: $\mathrm{X}_{2} \mathrm{Y}_{2}$ is planar. The fragment velocity distribution is narrow, and the spectral resolution is high). $\vec{k}_{p}$ and $\vec{k}_{a}$ describe the direction of the photolysis and analysis lasers for the case when curve (b) is obtained. $\vec{E} p$ is the polarization direction of the photolysis laser. Curve (c) is the result if $\vec{k}_{a}$ is pointing vertically to the $\mathrm{X}_{2} \mathrm{Y}_{2}$ plane. Figure $1 \mathrm{~b}$ contains in addition the fragment rotation indicated by $\vec{J}$. In order to obtain the information on the $\langle\vec{\mu} \vec{\jmath}\rangle$ correlation, the analysis laser needs also to be linearly polarized, as shown by the field vector $\vec{E} a$. In the case of $\vec{E} a$ being directed parallel to the fragment $\vec{J}$ vector, Q absorption lines will be observed, $R$ - and $P$ - lines cannot be excited at all. If $\vec{E} a$ is in the plane of the molecule the situation is reversed.

When the parent molecule rotates due to its thermal energy content its primary 
1

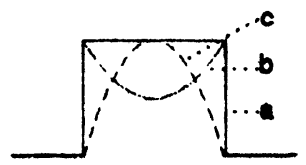

$$
\mathrm{I}\left(\mathrm{x}_{\mathrm{D}}\right) \sim \frac{1}{\Delta \nu_{\mathrm{D}}}\left[1+\beta \mathrm{P}_{2}\left(\mathrm{x}_{\mathrm{D}}\right)\right] ; \quad \mathrm{x}_{\mathrm{D}}=\frac{v-\nu_{0}}{\Delta \nu_{\mathrm{D}}}
$$

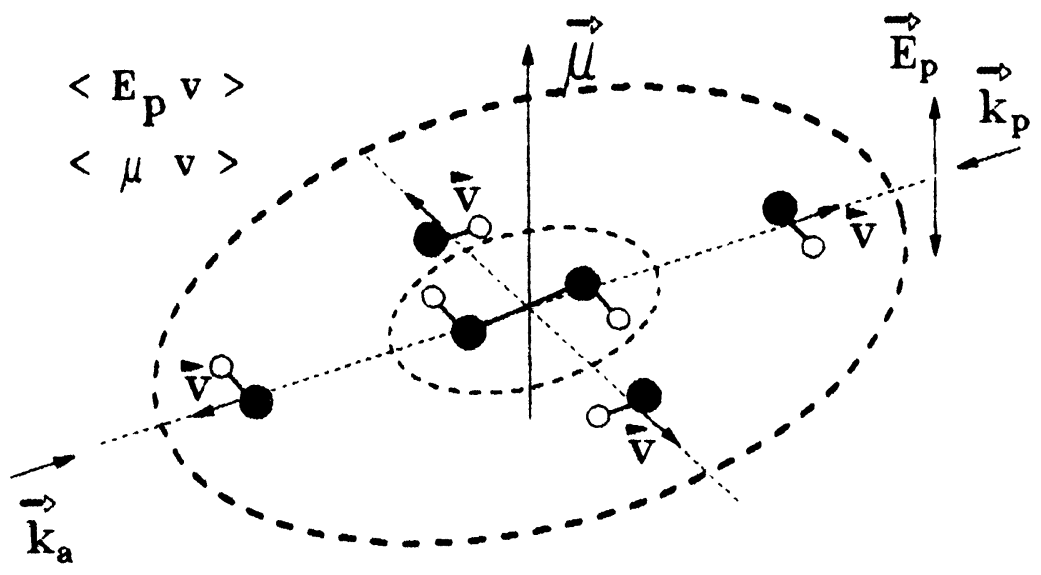

a)

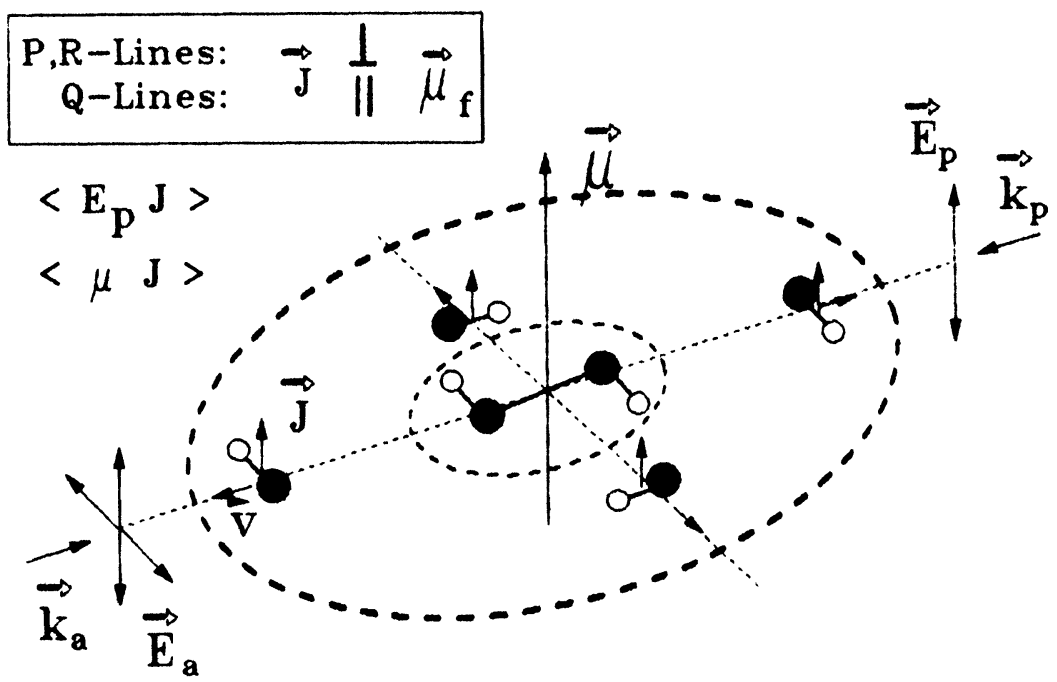

Figure 1 Pictorial view of vector correlations; (a) $\langle\mu v\rangle$ correlation; (b) $\langle\mu \mathrm{J}\rangle$ correlation the index prefers to the photolysis laser, the index (a) refers to the analysis laser.

alignment due to photon absorption is only existent for a time interval much shorter than the rotational period. This molecular rotation will lead to a smearing out of the line profiles deviating from those shown in Figure 1. Knowing the direction of $\vec{\mu}$ this deviation from the theoretical profile is a means to deduce the lifetime of the dissociating molecule. The method of indirectly extracted dissociation lifetimes has its special potential in the femtosecond time regime. 
Due to the primary excitation, $\vec{\mu}$ is correlated to the laboratory frame. In contrast, $\vec{v}$ and $\vec{J}$ are correlated to the molecular frame. Correlating $\vec{v}$ and $\vec{J}$ with each other leads to another vector correlation, $\langle\vec{v} \overrightarrow{\mathbf{J}}\rangle$, which contains only vector properties based on the molecular frame. This new correlation opens the possibility to even study dissociation processes of molecule exhibiting a long dissociation lifetime because the correlation depends no longer on this lifetime. We have carried out a transformation from the laboratory-based frame to the rotating molecular frame. As we have already explained, a more parallel or a more vertical correlation of these two vector properties directly reflect planar of torsional type of motion as a consequence of the forces which cause the dissociation.

For the case of $\mathrm{H}_{2} \mathrm{O}_{2}$ for instance the positive value of the $\langle\vec{v} \vec{J}\rangle$ correlation points to a strong dependence of the upper potential energy surface on the dihedral angle. $A b$ initio calculations of this surface directly prove this conclusion. ${ }^{1}$ The observed torsional dependence is in complete quantitative agreement with calculations of the dissociation dynamics using this Potential Energy Surface (PES).

As a direct consequence of the strong torsional dependence of the PES the two OH fragments which are formed as a twin from one original parent molecule must be found rotating opposite to each other with the same rotational energy. This new correlation - the coincident pair correlation-can be expressed as $\langle\overrightarrow{\mathrm{J}} \overrightarrow{\mathrm{J}}\rangle$ correlation.

How do we get this correlation from the experiment? Let us assume that there exist three different dissociation channels upon excitation:

$$
\mathrm{H}_{2} \mathrm{O}_{2}^{*} \longrightarrow \mathrm{OH}^{1}\left(J_{i}\right)+\left\{\begin{array}{l}
\mathrm{OH}^{2}\left(J_{i}-i\right)+\Delta E_{\mathrm{kin}}(i, i-1) \\
\mathrm{OH}^{2}\left(J_{i}\right)+\Delta E_{\mathrm{kin}}(i, i) \\
\mathrm{OH}^{2}\left(J_{i}+i\right)+\Delta E_{\mathrm{kin}}\left(i_{i} i+1\right)
\end{array}\right.
$$

$\mathrm{OH}^{1}\left(J_{i}\right)$ is the fragment which is observed because the analysis laser is set to the rotational transition with quantum number $J_{i}$. Its Doppler width is determined by the kinetic energy release. The three channels differ by their $\Delta E_{\text {kin }}$, giving rise to three different Doppler widths. As only $\mathrm{OH}^{1}\left(J_{i}\right)$ is detected, its line is a superposition of lines from the three dissociation channels exhibiting three different line widths (Figure 2). The step height is a quantitative measure of the contribution of the respective channel to the total dissociation. The experimental conditions for the absorption of a $\langle\overrightarrow{\mathrm{J}} \overrightarrow{\mathrm{J}}\rangle$ correlation is a spectral resolution of about $50 \mathrm{MHz}$ for the case of $\mathrm{OH}$ at $\mathbf{J} \approx 12 \hbar$.

It is a result of our observations that, at different dissociation wavelengths, there exists approximately a one to one correlation showing that the positive $\langle\vec{v} \vec{\jmath}\rangle$ correlation is fully proved: the rotation of the two fragments from the same dissociation is largely the result of a torsional motion of the parent during fragmentation. $^{2}$

In the fragmentation of hydrazoic acid, $\mathrm{HN}_{3}$, where the two diatomics $\mathrm{HN}$ and $\mathrm{N}_{2}$ are formed, the $\mathrm{N}_{2}$ fragment which is hard to detect by laser induced fluorescence can fully be determined by its partner molecule NH. Again, the results of correlation studies are in very good agreement with ab initio calculations of PES. ${ }^{3,4}$ As the dissociation follows from motions in different planes, the problem is rather complex. Besides, for the very low excitation energy at the dissociation limit, $\mathrm{N}_{2}$ is the main 


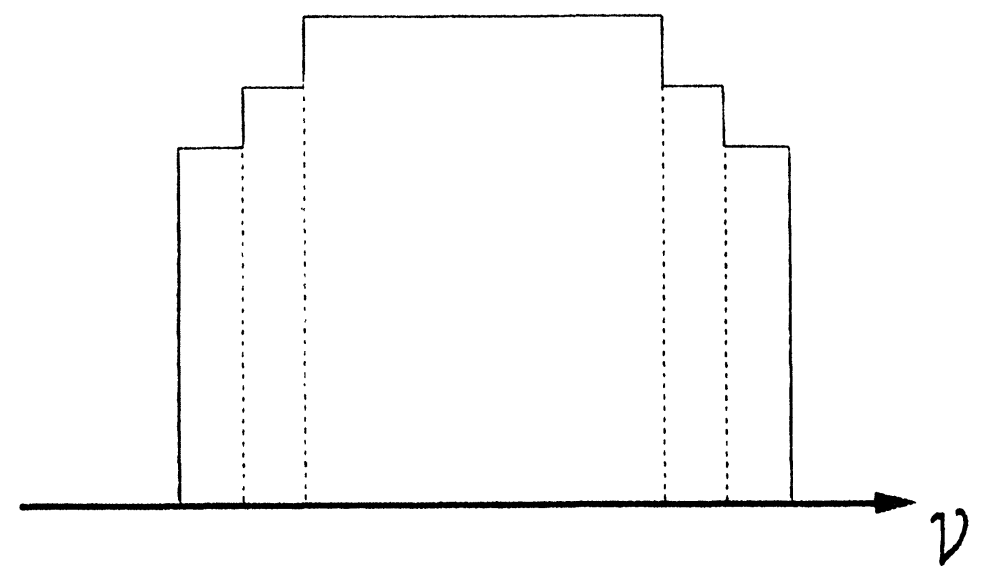

Figure 2 Coincident pair correlation. Schematic of line shape, simulating conditions of Eqs. 5a-5c.

acceptor of the excess energy showing the $\mathrm{HN}_{3}$ in plane $\left(v_{5}\right)$ and the out of plane $\left(v_{6}\right)$ bending motion to be most important. As a consequence $\mathrm{N}_{2}$ is found to be highly rotationally excited, which is not the case for NH due to its heavy-light character. The two different $\mathrm{NH} \Delta$-states show different behaviour which is further supported by a remarkable isotope effect.

Although in many of the experiments the parent molecules were prepared as cold molecules by supersonic jet expansion the excitation can only approximately be described as state-to-state selective. Further experiments from this laboratory will therefore use ir absorption in the first step to selectively prepare the parent molecules.

\section{References}

1. R. Schinke and V. Staemmler, Chem. Phys Lett. 145, 486 (1988).

2. K.-H. Gericke, H. G. Gläser, C. Maul and F. J. Comes, J. Chem. Phys. 92, 411 (1990).

3. K.-H. Gericke, T. Haas, M. Lock, R. Theinl and F. J. Comes, J. Phys. Chem. 95 (1991).

4. U. Meier and V. Staemmler, J. Phys. Chem. 95 (1991). 